\title{
MISCETLANEA
GEOGRAPIICA
}

WA R S A W A 1988

Urszula Kossowska-Cezak

\section{L'INFLUENCE DE L'HABITAT URBAIN SUR LA VARIABILITE DE LA TEMPERATURE DU JOUR AU LENDEMAIN}

L'étude de la variabilité de la température de l'air d'un jour à l'autre permet de constater la dépendance de celle-ci de la circulation atmosphérique, par contre, on ne prête que peu d'attention à l'influence de facteurs locaux. Une telle attitude paraît non fondée, car dans le cas de climats locaux dont les conditions thermiques sont considérablement modifiées, on pourrait s'attendre plutôt à ce que la variabilité de la température du jour au lendemain se forme différemment, par rapport aux terrains voisins. Prenons, à titre d'exemple, le climat urbain - variante d'un climat local ayant des conditions thermiques spécifiques. De nombreuses stations météorologiques dont les observations servent à diverses études, entre autres à celle de la variabilité de la température, sont. installées dans les villes. Ainsi, on est amene à se poser la question suivante: une agglomération influence-t-elle, et de quelle manière les variations de la température d'un jour à l'autre.

Afin de résoudre ce problème, on a effectué une analyse des valeurs de la température moyenne diurne d'un jour à l'autre, maximale et minimale, pour une période de cinq ans (1961-1965), valeurs fournies par cinq stations installées à Varsovie (fig. 1). Ces stations représentent les conditions suivantes: Université - habitat dense, au centre de la ville, Observatoire astronomique - parc assez vaste au centre de la ville, quartier de Bielany - habitat dispersé dans la partie nord de la ville, Station des pompes fluviales (sur la Vistule) - habitat dispersé dans la vallée de la Vistule, quartier d'Okęcie - terrains sans habitations (aéroport) aux confins sud-ouest de la ville.

L'analyse des valeurs moyennes mensuelles et annuelles de la variabilité de la température d'un jour à l'autre (table 1) a démontré que la température moyenne diurne se caractérise par la plus faible variabilité tandis que la température maximale - par la variabilité la plus haute. Une exception en est la station d'Okęcie - située dane un terrain sans habitations - où la plus haute variabilité accompagne la température minimale. La plus faible différence entre les stations se rapporte à la va- 


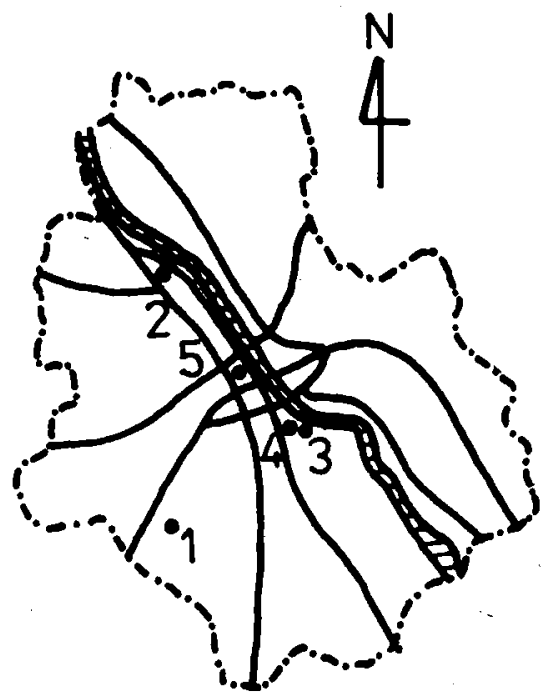

Fig. 1. Localisation des stations météorologiques à Varsovie

1 - Okęcie, 2 - Bielany, 3 - Station de Pompes fluviales (sur la Vistule), 4 - Observatoire astronomique, 5 - Université

Table 1

Les valeurs moyennes de la variabilité de la température d'un jour à l'autre $\left({ }^{\circ} \mathrm{C}\right)$ à Varsovie (1961-1965)

\begin{tabular}{l|l|l|c|c|c}
\hline \multirow{2}{*}{$\begin{array}{l}\text { Varia- } \\
\text { bilité }\end{array}$} & Okecie & Bielany & $\begin{array}{c}\text { Station } \\
\text { des } \\
\text { Pompes }\end{array}$ & $\begin{array}{l}\text { Observa- } \\
\text { toire }\end{array}$ & $\begin{array}{c}\text { Univer- } \\
\text { sitć }\end{array}$ \\
\cline { 2 - 6 } & & & & & \\
$\mathrm{t}$ & 2,03 & 2,04 & 1,97 & 1,92 & 1,94 \\
$\mathrm{t}_{\max }$ & 2,35 & 2,39 & 2,37 & 2,34 & 2,29 \\
$\mathrm{t}_{\min }$ & 2,40 & 2,23 & 2,23 & 2,12 & 2,05 \\
\hline
\end{tabular}

riabilité de la température maximale. la plus grande - à la variabilité de la température minimale. Dans les quartiers du centre, surtout là, où l'habitat est dense, la variabilité de la température minimale diminue nettement (fig. 2); cette observation se rapporte également, à un certain degré, à la température moyenne diurne.

La variabilité de la température dans les quartiers du centre n'est pas seulement plus faible, du point de vue genéral, mais également ses courses annuelles sont différentes. Aux confins de la ville, les variations les plus élevées de la température moyenne diurne apparaisschnt en hiver (décembre, janvier), c'est-à-dire au cours de la période des plus grandes variations de la température minimale, tandis que dans les quartiers du centre - au début de l'été (juin), autrement dit durant la période des variations les plus élevées de la température maximale. La variabilité du 


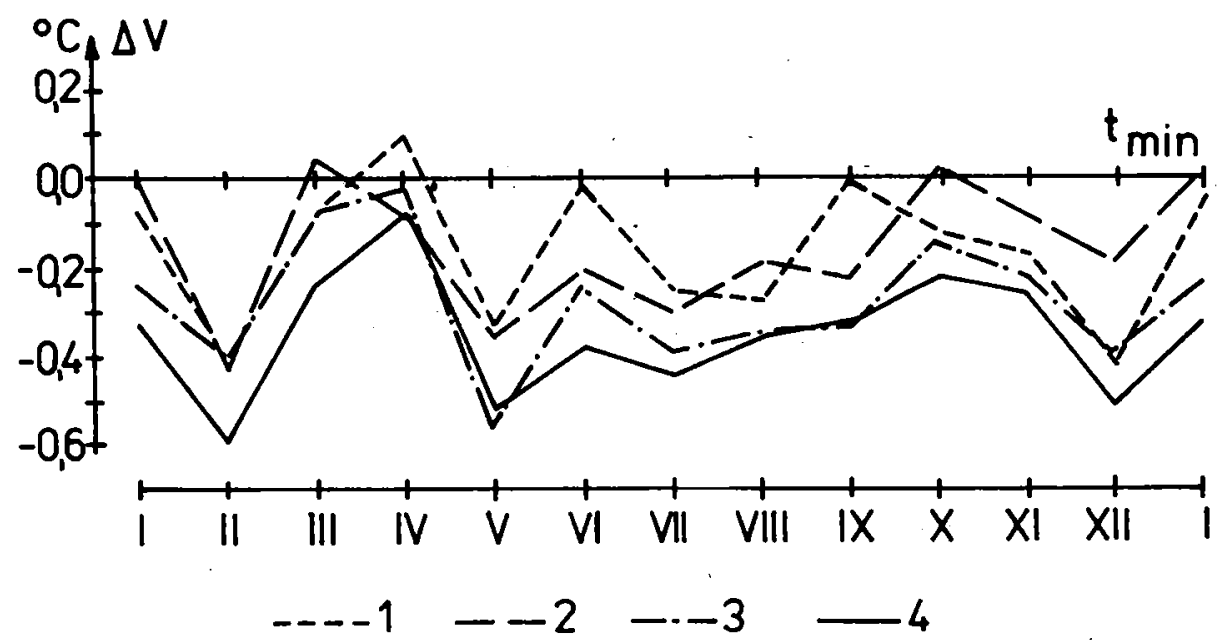

Fig. 2. Différences de la variabilité de la température minimale du jour au lendemain entre les stations installées dans des terrains habités et la station d'Okęcie observées au cours d'une année. 1 - Bielany, 2 - Station des pompes fluviales, 3 - Observatoire astronomique, 4 - Université

jour au lendemain de la température moyenne diurne dans les quartiers du centre est plus faible qu'aux confins, surtout en automne et en hiver; cette même variabilité concernant la température maximale est légèrement plus faible au cours de l'année à l'exception du début de l'été, et pour la température minimale - plus faible pendant toute l'année.

$\mathrm{La}$ fréquence des variations dont les valeurs et les directions sont différentes se ressemble dans toutes les stations en ce qui concerne la température aussi bien moyenne diurne que maximale, par contre cette fréquence est différenciée dans le cas de la température minimale. Dans les stations du centre - Université et Observatoire - le pourcentage de variations inférieures à $2{ }^{\circ} \mathrm{C}$ (aussi bien les hausses que les baisses) dépasse de $2-3 \%$ $(5-6 \%$ au total) celui des variations des températures aux confins, par contre le pourcentage de variations dépassant $4^{\circ} \mathrm{C}$ est proportionnellement plus faible. Le nombre de jours où les variations de la température minimale dépassent $8 \mathrm{C}$ se présente comme suit (pour la période étudiée de cinq ans): Okęcie (aéroport) - 41 jours, Bielany - 28 jours, Station des pompes -26 jours, Observatoire -27 , et Université - seulement 22 jours.

Le pourcentage de baisses de la température minimale observées en stations les plus éloignées du centre de la ville, à savoir Okęcie et Bielany dépasse de $1 \%$ celui des stations du centre (Université et Observatoire).

La valeur moyenne de la variabilité de la température au cours des mois choisis de la période étudiée de cinq ans changeait dans de larges limites, de même que la valeur de la différenciation de la variabilité de la température entre diverses stations. Afin de déterminer les conditions où l'on observe l'apparition ou la disparition de grandes différences entre 
les stations, on a examiné les températures au cours de sept mois particuliers. On a choisi quatre mois à la plus haute variabilite (decembre 1961, janvier et février 1963, avril 1961) et trois mois à plus faible variabilité (février 1961, octobre 1962 et mars i964). La table 2 présente les valeurs de la variabilité moyenne mensuelle pour trois des sept mois choisis.

Table 2

Les valeurs moyennes de la variabilité de la température d'un jour à l'autre $\left({ }^{\circ} \mathrm{C}\right)$ à Varsovie, au cours des mois à la plus haute (A) et à la plus faible (B) variabilité, en 1961-1965

\begin{tabular}{l|l|l|l|c|c|c}
\hline \multirow{2}{*}{ Mois } & \multirow{2}{*}{$\begin{array}{c}\text { Varia- } \\
\text { bilité }\end{array}$} & Okęcie & Bielany & $\begin{array}{c}\text { Station } \\
\text { des } \\
\text { Pompes }\end{array}$ & $\begin{array}{c}\text { Observa- } \\
\text { toire }\end{array}$ & $\begin{array}{c}\text { Univer- } \\
\text { sité }\end{array}$ \\
\hline
\end{tabular}

\begin{tabular}{|c|c|c|c|c|c|c|}
\hline A & & & & & & \\
\hline Décembre & $\overline{\mathbf{t}}$ & 3,50 & 3,43 & 3,46 & 3,36 & 3,32 \\
\hline 1961 & $t_{\max }$ & 3,11 & 3,19 & 3,19 & 3,02 & 3,25 \\
\hline & $t_{\min }$ & 3,79 & 3,46 & 3,40 & 3,32 & 3,22 \\
\hline A & & & & & & \\
\hline Février & $\overline{\mathrm{t}}$ & 2,82 & 2,15 & 2,31 & 2,10 & 2,00 \\
\hline 1963 & $t_{\max }$ & 1,67 & 1,71 & 1,68 & 1,34 & 1,48 \\
\hline & $t_{\min }$ & 4,76 & 3,25 & 3,61 & 3,38 & 3,24 \\
\hline $\mathbf{B}$ & & & & & & \\
\hline Février & $\overline{\mathbf{t}}$ & 1,04 & 1,25 & 1,29 & 1,32 & 1,30 \\
\hline 1961 & $t_{\max }$ & 1,76 & 1,78 & 1,75 & 1,71 & 1,70 \\
\hline & $t_{\min }$ & 1,41 & 1,38 & 1,30 & 1,32 & 1,32 \\
\hline
\end{tabular}

Une analyse des valeurs journalières de la température moyenne diurne maximale et minimale, confrontées avec des types de la circulation atmosphérique au-dessus de l'Europe Centrale (Osuchowska-Klein, 1978) et avec les données sur la nébulosité et les vents à Varsovie, a démontré que la variabilité de la température d'un jour à l'autre genéralement-haute ou généralement faible dépend du caractère de la circulation atmosphérique. Une alternance de types de circulation de différents secteurs, avec un apport elevé des types anticycloniques est à l'origine des variations importantes de la température, tandis que la prédominance de la circulation d'un seul secteur donne une température uniforme pendant plusieurs jours donc favorise une variabilité peu élevée. Par contre, la grandeur de la différenciation de la variabilité de la température entre les stations dépend des valeurs de la nébulosité, ainsi que de la direction et de la vitesse des vents. Etant donné que les éléments météorologiques cités plus haut influent, de façon considérable, sur les valeurs locales du minimum journalier, la variabilité de la température minimale s'étant révélée sujette à la plus grande différenciation en fonction du caractère de l'habitat et de la situation par rapport au centre de la ville, fera lobjet de notre attention. 


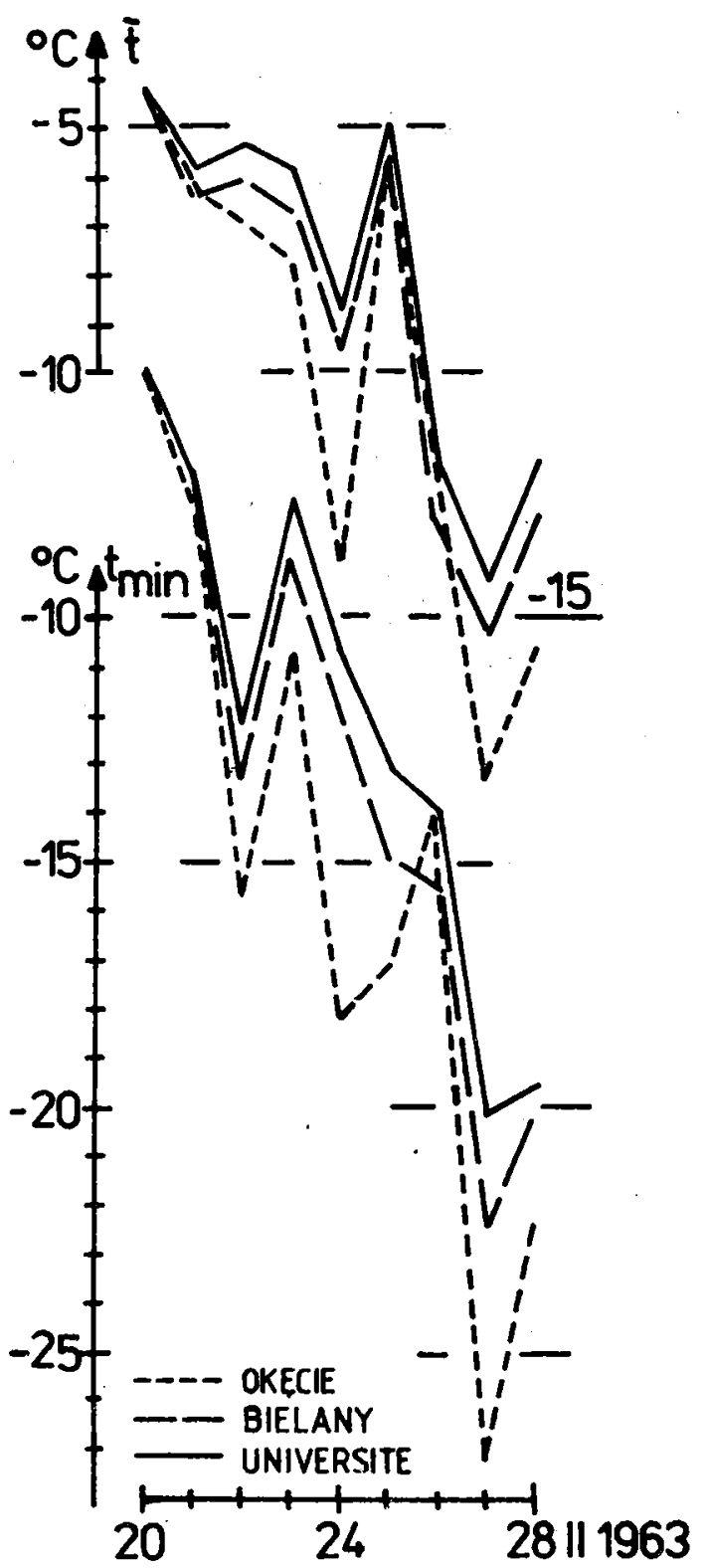

Fig. 3. Les temperatures moyennes diurne et minimale observees du 20 au 28 février 1963 
On a constaté qu'une faible nébulosité le nuit (surtout au cours de la période froide où la nuit est la plus longue) favorise d'importantes baisses de la température - plus grandes dans un terrain découvert que dans un terrain habité. Dans le cas d'une importante nébulosité nocturne succédant à une nuit claire, la température minimale en dehors des quartiers habités est légèrement plus basse par rapport à la température dans les quartiers du centre - il en résulte que la hausse de la température par rapport au jour précédent se révèle nettement plus grande en dehors de la ville. De telles situations avaient lieu fréquemment, entre autres en décembre 1961 et en février 1963 - mois s'étant distingués par une grande variabilité de la température (table 2). Les différences particulièrement importantes de la température minimale et, par là-même, de son variabilité du jour au lendemain, entre les quartiers du centre et les confins de Varsovie, ont eu lieu dans la dernière dizaine du février 1963 (table 3). Les nuits du 23 au 24 et du 26 au 27 février - avec le ciel clair et le vent faible, celles du 24 au 25 , et du 25 au 26 février, par contre, avec le ciel nuageux et les vents forts; d'importantes baisses de la température minimale observées en station d'Okęcie étaient accompagnées d'une baisse importante de la température moyenne diurne.

L'analyse des valeurs journalières de la température relevées en cinq stations météorologiques de Varsovie a démontré que les plus importantes baisses de la température se manifestent dans la partie de la ville exposée aux vents. Comme Okęcie est non seulement le quartier le plus éloigné de l'agglomération mais aussi situé du côté des vents prédominants (d'ouest et de sud-ouest), la variabilité de la température, surtout minimale, est plus grande qu'en d'autres stations. Cependant, les jours avec les vents du nord ou du nord-est, la température peut être pareille à celle du centre, par contre on observe une baisse considérable dans le quartier de Bielany, situé dans le nord de la ville. Une telle situation a eu lieu,. entre autres, le matin du 26 février 1963 où à Bielany et même dans les quartiers du centre on a noté une baisse de la température minimale par rapport au jour précédent, tandis que la station d'Okęcie a observé une hausse de la température. Les jours de calme plat, d'importantes baisses de la température se manifestent dans la dépression de la vallée de la Vistule (Station des pompes).

Une plus grande variabilité de la température dans le terrain habité observée au printemps et au début de l'été dépend des jours de beaú temps typiques pour cette période de l'année. La température s'élève alors au milieu des habitations, entre autres du fait de l'affaiblissement du mouvement de l'air. Des situations pareilles peuvent avoir lieu également les jours particuliers d'autres saisons de l'année.

Une faible variabilité de la température d'un jour à l'autre se manifeste dans les mois, où, jour après jour, l'air peu différencié au point de vue thermique afflue d'un seul secteur. Lorsqu'une telle situation est accompagnée 
d'un ciel couvert et des vents forts ou modérés, les variations de la température dans la partie de la ville exposée aux vents peuvent être inférieures à celles observées dans les quartiers du centre, comme c'était le cas en février 1961 (table 2).

L'analyse des valeurs des moyennes aussi bien mensuelles que diurnes des mois choisis a démontré que l'habitat urbain exerce une influence sur la valeur de la variabilité de la température du jour au lendemain. Dans le cas de la variabilité de la température moyenne diurne et de la température maximale, cette influence est insignifiante et pourrait même être passée sous silence dans des comptes rendus basés sur les données fournies par les stations installées dans divers sites. Par contre, cette influence apparaît nettement dans le cas de la température minimale et se manifeste sous forme d'une diminution de sa variabilité, en comparaison avec le terrain .ouvert. Ce phénomène est facile à expliquer, car l'habitat urbain modifie avec le plus d'intensité les valeurs d'un minimum diurne de la température (Kossowska, 1973) en contribuant ainsi à une augmentation de ces valeurs. Ce fait devrait être pris en considération lors d'une interprétation des résultats obtenus des stations urbaines; il paraît même que la valeur de la variabilité de la température minimale d'un jour à l'autre devrait devenir une des caractéristiques thermiques du climat de la ville et, avec la valeur de la température minimale, être examinée par rapport à une valeur correspondante représentant un terrain en dehors de la ville.

\section{BIBLIOGRAPHIE}

Koss owska, U., 1973, „Osobliwości klimatu miejskiego na przykladzie Warszawy”

(Particularités du climat urbain - le cas de Varsovie), Prace $i$ Studia IG UW, vol. 12. O s u c h ow s k a-K 1 e i n, B., 1978, Katalog typów cyrkulacji atmosferycznej (Le Catalogue de types de circulation atmosphérique), WKit, Warszawa. 
